\title{
Spin-dependent transport for armchair-edge graphene nanoribbons between ferromagnetic leads
}

\author{
Benhu Zhou, ${ }^{1}$ Xiongwen Chen, ${ }^{1}$ Benliang Zhou, ${ }^{1}$ Kai-He Ding, ${ }^{2}$ and Guanghui Zhou ${ }^{1},{ }^{2}$ \\ ${ }^{1}$ Department of Physics and Key Laboratory for Low-Dimensional Structures and Quantum \\ Manipulation (Ministry of Education), Hunan Normal University, Changsha 410081, China \\ ${ }^{2}$ Department of Physics and Electronic Science, Changsha University of Science and Technology, Changsha 410076, China and \\ ${ }^{3}$ International Center for Materials Physics, Chinese Academy of Sciences, Shenyang 110015, China
}

\begin{abstract}
We theoretically investigate the spin-dependent transport for the system of an armchair-edge graphene nanoribbon (AGNR) between two ferromagnetic (FM) leads with arbitrary polarization directions at low temperatures, where a magnetic insulator is deposited on the AGNR to induce an exchange splitting between spin-up and -down carriers. By using the standard nonequilibrium Green's function (NGF) technique, it is demonstrated that, the spin-resolved transport property for the system depends sensitively on both the width of AGNR and the polarization strength of FM leads. The tunneling magnetoresistance (TMR) around zero bias voltage possesses a pronounced plateau structure for system with semiconducting 7-AGNR or metallic 8-AGNR in the absence of exchange splitting, but this plateau structure for 8-AGNR system is remarkably broader than that for 7-AGNR one. Interestingly, the increase of exchange splitting $\Delta$ suppresses the amplitude of the structure for 7-AGNR system. However, the TMR is enhanced much for 8-AGNR system under the bias amplitude comparable to splitting strength. Further, the current-induced spin transfer torque (STT) for 7-AGNR system is systematically larger than that for 8-AGNR one. The findings here suggest the design of GNR-based spintronic devices by using a metallic AGNR, but it is more favorable to fabricate a current-controlled magnetic memory element by using a semiconducting AGNR.
\end{abstract}

PACS numbers: 73.63.-b, 85.35.-p, 72.25.Hg

\section{INTRODUCTION}

Spintronics utilizes the electron spin degree of freedom to carry information in electronic devices and functionalities. Its central task is to control electron spin for information storage and precessing. ${ }^{1}$ Graphene, a single layer of carbon, has low intrinsic spin-orbit and hyperfine couplings, ${ }^{2}$ long spin diffusion lengths $(\sim 2 \mu \mathrm{m}){ }^{3}$ These features suggest that graphene is a promising candidate for the potential applications in spintronics. Triggered by these findings, a large number of works have been devoted to the spin-dependent transport in graphene and graphene-FM heterostructures, as prototypical spintronic devices. For example, a graphene spin-valve device has been successfully fabricated showing that spin-polarized currents can be injected by means of magnetic (cobalt, permalloy, etc.) electrodes, ${ }^{4-9}$ which shows high spin-polarized injection efficiency. Meanwhile, a 10\% magnetoresistance (MR) is observed ${ }^{10}$ in a graphene contacted by two soft magnetic electrodes. The further experiments have found that a inserted tunnel film at the graphene/electrode interface favors MR which can reach up to $\sim 12 \% .^{11}$ Additionally, it is expected ${ }^{12}$ that the spin dependence of the electron energy can be also produced by a FM insulator substrate which acts as an effective magnetic field parallel to the graphene layer. ${ }^{13,14}$. Theoretically, using the tight-binding model, Brey and Fertig ${ }^{15}$ found that the MR is rather small due to the weak dependence of the graphene conductivity on the electronic parameters of FM leads. Using a continuous model by NGF, Ding et al. ${ }^{16}$ have demonstrated that the TMR exhibits a cusp around zero bias in the absence of external magnetic field for a similar device. Recently, special attentions have been paid to zigzagedge graphene nanoribbons (ZGNRs) spin-valve devices since ZGNRs exhibit fascinating phenomenon due to the size confinement and the edge state. ${ }^{17-19}$ For example, some theoret- ical studies have predicated that ZGNR-based spin-valve devices have a very large TMR. ${ }^{20-25}$

On the other hand, the leads (electrodes) for spin-valve devices in the previous works have been supposed to be twodimensional (of honeycomb- or square-lattice) type, but more realistically they should be modeled as three-dimensional semi-infinite slabs. Further, the investigation on the TMR for GNR-magnetic junctions is still lacking so far, especially, the influence of an exchange field on the spin-dependent transport. Another important phenomenon in spintronics is the reverse effect to TMR, i.e., spin-polarized electrons passing from the left FM layer into the right layer, where the magnetization deviates the left by an angle, may exert a torque to the right FM lead. This effect is the so-called ${ }^{26,27}$ STT which has been extensively studied both theoretically ${ }^{28-32}$ and experimentally ${ }^{33,34}$ for conventional magnetic junction systems. A demonstration of the spin-transfer phenomenon is the current-induced magnetic switching, which has been confirmed experimentally in spin-valves ${ }^{35,36}$ and magnetic tunnel junctions (MTJs) ${ }^{37}$ Thus, current-induced magnetic switching provides a powerful new tool for the study of spin transport in magnetic nanostructures. In addition, it offers the intriguing possibility of manipulating high-density nonvolatile magnetic-device elements, such as magnetoresistive random access memory (MRAM), without applying cumbersome magnetic fields. ${ }^{38}$ However, the previous works on spin-dependent transport through a graphene-based system are mainly focused on the tunnel current and the TMR effect. ${ }^{10-16,20-25}$ The investigation on the current-induced STT in such system is sparsely reported. Although we have addressed this issue for a FM/graphene/FM device, ${ }^{39}$ in this paper we extend it to a FM/AGNR/FM system.

In this paper, we present a theoretical investigation on the fully spin-dependent transport through an AGNR between two 


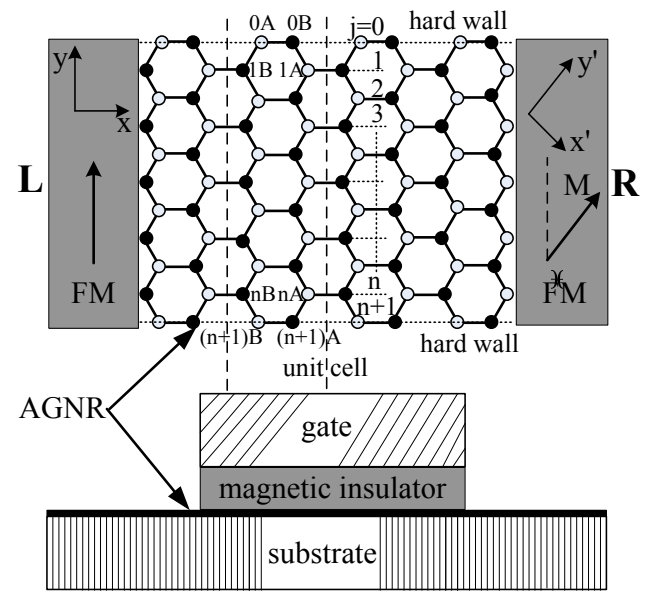

FIG. 1: Schematic illustration of the system consisting of an AGNR between two FM leads, where a FM insulator layer with a control gate is deposited on AGNR. A unit cell of AGNR represented by two dashed lines contains $n$ numbers of $A$ and $B$ sublattice sites labeled as $1 A, 1 B, \cdots, n A, n B$. Two additional hard walls are imposed on both edges at chains $j=0$ and $n+1$. The magnetic moment of the two leads is aligned at a relative angle $\theta$.

FM leads with arbitrary polarization direction at low temperatures, where a magnetic insulator is deposited on AGNRs to induce an exchange splitting. By using the Keldysh NGF method, ${ }^{40,41}$ the density of state (DOS), the linear conductance, the differential conductance, and the current for the system have been calculated separately for spin-up and -down channels, and consequently the TMR dependence on applied bias has been calculated. Further, the dependence of STT on the bias and the polarization angle of the two leads have also been examined. It is demonstrated that, the spin-resolved transport property of the system depends sensitively on both the widths of AGNRs and the polarization strength of FM leads. Around zero bias the TMR versus bias voltage possesses a pronounced plateau structure for both semiconducting 7-AGNR and metallic 8-AGNR systems without exchange splitting. The plateau structure for 8-AGNR system is remarkably broader than that for 7-AGNR one. Interestingly, the increase of the exchange splitting strength suppresses the amplitude of this structure for 7-AGNR system. However, the TMR is enhanced at bias that ranged from $-\Delta$ to $\Delta$ for 8 -AGNR system. Further, the current-induced STT for 7-AGNR system is systematically larger than that for 8-AGNR system.

The rest of the paper is organized as follows. In Sec. II, we derive the analytical expressions for the spin-dependent DOS, the conductance, the current, and the STT, starting from the system Hamiltonian by NGF approach. Some numerical examples and the discussions for the results are demonstrated in Sec. III. Finally, Sec. IV concludes the paper.

\section{MODEL AND METHOD}

The geometry of the system considered in this paper is shown in Fig. 1, where an AGNR contains two sublattices denoted by $A$ and $B$. We use $n$, the number of $A(B)$-site atoms in a unit cell, to denote AGNR with different width. Ideal (perfect) AGNRs with $n=3 j-1$ with positive integer $j$ are metallic, otherwise are semiconducting. ${ }^{18,19}$ From the top down, atoms in a unit cell are labeled as $1 A, 1 B, \cdots, n A, n B$. As shown in Fig. 1, the hard-wall condition is imposed on both edges at chains $j=0$ and $n+1$. The definition of GNRs in this work is in accord with the previous convention, ${ }^{19}$ i.e., an AGNR is identified by the number of carbon zigzag chains forming the width of ribbon, and an AGNR with $n$ carbon chains is named as $n$-AGNR, therefore its width is $W=(n-1) \sqrt{3} a / 2$, where $a=1.42 \AA$ is the $\mathrm{C}-\mathrm{C}$ bond length.

The system under consideration here is composed of a semiconducting 7-AGNR or a metallic 8-AGNR connected to two FM leads, which can be considered as a MTJ (or a spinvalve) device. A layer of magnetic insulator is deposited on the top of AGNR sample to induce an exchange splitting between spin-up and -down carriers. ${ }^{12}$ The gate allows us to control the Fermi level locally, i.e., to create a tunable barrier in AGNR. In this way, controlling on both charge and spin carrier concentrations can be achieved. The magnetic moment $M_{L}$ of the left FM lead is assumed to be parallel to the $y$-axis, while the moment $M_{R}$ of the right FM deviates from the $y$ direction by a relative angle $\theta$. The tunneling current flows along the $x$-axis [see Fig. 1]. We assume that the magnitude of exchange splitting can be modulated by the magnetic insulator. It seems a reasonable assumption that the magnetic insulator is made of different materials from FM leads.

The total Hamiltonian for the system considered reads

$$
H=H_{G}+H_{L}+H_{R}+H_{T},
$$

where $H_{G}$ describes the central AGNR region, $H_{L(R)}$ is the Hamiltonian for the left (right) FM lead, and $H_{T}$ for the coupling between AGNR and leads. Here, spin-orbit interaction in graphene or GNRs is neglected because it is too weak and is of the order of 3-4 meV, ${ }^{42,43}$ it opens up a gap of the order of $10^{-3} \mathrm{meV}$ at the Dirac point. In the tight-binding approximation, these partial Hamiltonians can be respectively written as following:

$$
H_{G}=\sum_{\beta i, \sigma}\left(\epsilon_{0}+s \Delta\right) a_{\beta i, \sigma}^{\dagger} a_{\beta i, \sigma}+t \sum_{<i j>, \sigma}\left(a_{A i, \sigma}^{\dagger} a_{B j, \sigma}+\text { H.c }\right),
$$

where $a_{\beta i, \sigma}^{\dagger}\left(a_{\beta i, \sigma}\right)$ creates (annihilates) an electron on site $i$ with spin $\sigma$ and sublattice index $\beta=A(B), \sigma=\uparrow(\downarrow)$ represents the spin-up (-down) state of electrons, $\epsilon_{0}$ is the on-site energy, $t(\approx 2.75 \mathrm{eV})$ the nearest-neighbor hopping energy, and $\Delta$ the exchange splitting energy induced by the FM insulator on the top of AGNR, $s= \pm 1$ stands for the electron spin parallel $(+)$ or antiparallel (-) to the exchange field. The sum over $\langle i, j\rangle$ is restricted to the nearest-neighbor atoms. Hamiltonian (2) can also be rewritten in momentum space

$H_{G}=\sum_{\beta, \mathbf{q} \sigma}\left(\epsilon_{0}+s \Delta\right) a_{\beta, \mathbf{q}}^{\dagger} a_{\beta, \mathbf{q}}+\sum_{\mathbf{q} \sigma}\left[\phi(\mathbf{q}) a_{A, \mathbf{q} \sigma}^{\dagger} a_{B, \mathbf{q} \sigma}+\right.$ H.c. $],(3)$

where $\phi(\mathbf{q})=-t\left[2 e^{i q_{x} a / 2} \cos \left(\frac{\sqrt{3} a}{2} q_{y}\right)+e^{-i q_{x} a}\right]$ is the structural factor with wave-vector in the $x$-direction within the first Brillouin zone $\left(0 \leq\left|q_{x}\right| \leq \pi /(3 a)\right)$ and the discretized wave-vector in the $y$-direction $q_{y}=\frac{2}{\sqrt{3} a} \frac{m \pi}{n+1}(m=1,2, \cdots, n)$. 
The two Hamiltonians

$$
H_{L}=\sum_{\mathbf{k}, \sigma} \epsilon_{\mathbf{k} L \sigma} c_{\mathbf{k} L \sigma}^{\dagger} c_{\mathbf{k} L \sigma}
$$

and

$H_{R}=\sum_{\mathbf{k}, \sigma}\left[\epsilon_{R}(\mathbf{k})-\sigma M_{R} \cos \theta\right] c_{\mathbf{k} R \sigma}^{\dagger} c_{\mathbf{k} R \sigma}-M_{R} \sin \theta c_{\mathbf{k} R \sigma}^{\dagger}{ }^{c} \mathbf{k}_{\mathbf{k} \bar{\sigma}}(5)$

are respectively for the left and right lead, where $\epsilon_{\mathbf{k} L \sigma}=\epsilon_{L}(\mathbf{k})-$ $e V-\sigma M_{L}$ with applied bias voltage $V$ and magnetic momentum $M_{L}, \epsilon_{L,(R)}(\mathbf{k})$ is the single-particle dispersion for the left (right) lead, $c_{\mathbf{k} L(R) \sigma}^{\dagger}\left(c_{\mathbf{k} L(R) \sigma}\right)$ is the creation (annihilation) operator of an electron with wavevector $\mathbf{k}$ in the left (right) lead, $\bar{\sigma}$ denotes the opposite spin-polarization with respect to $\sigma$. Note that here the coupling between the left lead and AGNR involves the $B$ sublattice, while it between the right lead and AGNR involves the $A$ sublattice (see Fig. 1). However, Hamiltonian (5) will be slightly different if the coupling at two interfaces involves the same sublattice, but the result is unchanged.

The coupling Hamiltonian

$$
\begin{aligned}
H_{T}= & \frac{1}{\sqrt{N}} \sum_{\mathbf{k} L \mathbf{q} \sigma}\left(T_{\mathbf{k} L \mathbf{q}} c_{\mathbf{k} L \sigma}^{\dagger} a_{A, \mathbf{q} \sigma}+\text { H.c. }\right) \\
& +\frac{1}{\sqrt{N}} \sum_{\mathbf{k} R \mathbf{q} \sigma}\left(T_{\mathbf{k} R \mathbf{q}} c_{\mathbf{k} R \sigma}^{\dagger} a_{B, \mathbf{q} \sigma}+\text { H.c. }\right),
\end{aligned}
$$

where $T_{\mathbf{k} L \mathbf{q}}\left(T_{\mathbf{k} R \mathbf{q}}\right)$ is the coupling matrix between left (right) lead and AGNR, $N$ is the number of sites in sublattice $A$ or $B$. It should be pointed out that the annihilation operator $a_{B, \mathbf{q}}$ in Eq. (6) should be replaced by $a_{A, \mathbf{q} \sigma}$ if the coupling between right lead and AGNR involves the $A$ sublattice.

Now we employ the usually defined retarded, advanced and correlated Green's function (GF $)^{40,41}$ $G_{\mathbf{q}^{\prime}, \mathbf{q}^{\prime} a}^{\sigma \sigma^{\prime}, r}\left(t_{2}, t_{1}\right)=-i \theta\left(t_{2}-t_{1}\right)\left\langle\left\{a_{\mathbf{q} \sigma}\left(t_{2}\right), a_{\mathbf{q}^{\prime} \sigma^{\prime}}^{\dagger}\left(t_{1}\right)\right\}\right\rangle$, $G_{\mathbf{q}^{a}, \mathbf{q}^{\prime} a}^{\sigma \sigma^{\prime}, a}\left(t_{2}, t_{1}\right)=i \theta\left(-t_{2}+t_{1}\right)\left\langle\left\{a_{\mathbf{q} \sigma}\left(t_{2}\right), a_{\mathbf{q}^{\prime} \sigma^{\prime}}^{\dagger}\left(t_{1}\right)\right\}\right\rangle, \quad$ and $G_{\mathbf{q}^{\prime}, \mathbf{q}^{\prime} a}^{\sigma \sigma^{\prime}<}\left(t_{2}, t_{1}\right)=i\left\langle a_{\mathbf{q}^{\prime} \sigma^{\prime}}^{\dagger}\left(t_{1}\right), a_{\mathbf{q} \sigma}\left(t_{2}\right)\right\rangle$ to the total Hamiltonian in spin space. When the unperturbed GF $g_{\mathbf{q} a, \mathbf{q} a}^{r, a}(\omega)$ of the AGNR is obtained, one can obtain $G_{\mathbf{q}_{a}, \mathbf{q}^{\prime} a}^{r, a}(\omega)$ and $G_{\mathbf{q} a, \mathbf{q}^{\prime} a}^{<}(\omega)$ from the standard GF technique and the Keldysh equation $^{40}$

$$
G_{\mathbf{q}_{a, \mathbf{q}^{\prime} a}^{r, a}}^{r a}(\omega)=\delta_{\mathbf{q} \mathbf{q}^{\prime}} g^{r, a} \mathbf{q} a, \mathbf{q} a_{a}^{r}(\omega)+g_{\mathbf{q} a, \mathbf{q}_{a}}^{r, a}(\omega) T^{r, a}(\omega) g_{\mathbf{q}^{\prime} a, \mathbf{q}^{\prime} a}^{r, a}(\omega),(7)
$$

$$
G_{\mathbf{q} a, \mathbf{q}^{\prime} a}^{<}(\omega)=G_{\mathbf{q}^{\prime}, \mathbf{q}^{\prime} a}^{r}(\omega) \Sigma^{<}(\omega) G_{\mathbf{q} a, \mathbf{q}^{\prime} a}^{a}(\omega),
$$

where $T^{r, a}(\omega)=\Sigma^{r, a}(\omega) /\left[1-g_{a a}^{r, a}(\omega) \Sigma^{r, a}(\omega)\right]$ with $g_{a a}^{r, a}(\omega)=$ $1 / N \sum_{\mathbf{q}} g_{\mathbf{q} a, \mathbf{q} a}^{r, a}(\omega)$ and $\Sigma^{r, a}(\omega)=\mp i / 2\left[\boldsymbol{\Gamma}_{L \mathbf{q q}} \mathbf{q}^{\prime}(\omega)+R \boldsymbol{\Gamma}_{R \mathbf{q q}} \mathbf{q}^{\dagger}\right]$ is the retarded/advanced self-energy, and $\Sigma^{<}(\omega)=$ $i\left[f_{L}(\omega) \boldsymbol{\Gamma}_{L \mathbf{q} \mathbf{q}^{\prime}}(\omega)+f_{R}(\omega) R \boldsymbol{\Gamma}_{R \mathbf{q q}} R^{\dagger}\right]$ is the correlated self-energy. In the above equations, the matrix

$$
R=\left(\begin{array}{cc}
\cos (\theta / 2) & -\sin (\theta / 2) \\
\sin (\theta / 2) & \cos (\theta / 2)
\end{array}\right),
$$

$f_{L(R)}(\omega)=1 /\left[e^{\left(\omega-\mu_{L R}\right) / k_{B} T}+1\right]$ is the Fermi-Dirac distribution function of the left (right) FM lead, the linewidth function

$$
\boldsymbol{\Gamma}_{\alpha \mathbf{q q}^{\prime}}(\omega)=\left(\begin{array}{cc}
\boldsymbol{\Gamma}_{\alpha \mathbf{q q}^{\prime} \uparrow}(\omega) & 0 \\
0 & \boldsymbol{\Gamma}_{\alpha} \mathbf{q} \mathbf{q}^{\prime} \downarrow \\
& (\omega)
\end{array}\right)
$$

with $\Gamma_{\alpha \mathbf{q q}^{\prime}}^{\sigma}(\omega)=2 \pi \sum_{\mathbf{k}} T_{\mathbf{k} \alpha \mathbf{q}}^{*} T_{\mathbf{k} \alpha \mathbf{q}^{\prime}} \delta\left(\omega-\varepsilon_{\mathbf{k} \alpha \sigma}\right)$ describes the influence of the leads. Here, we consider electrons near the Fermi level $E_{F}$ which contribute predominantly to the tunneling. In this case one may assume that the coupling matrix is independent of energy and set $\boldsymbol{\Gamma}_{\alpha \mathbf{q} \mathbf{q}^{\prime}}^{\sigma}=\boldsymbol{\Gamma}_{\alpha}^{\sigma}$.

Furthermore, the isolated retarded GF for an AGNR is defined as

$$
g_{a a}^{r}(\omega)=\sum_{q_{x}, q_{y}, \pm} \frac{|\psi\rangle_{ \pm \pm}\langle\psi|}{\left[\omega-\left(\epsilon_{0}+s \Delta\right)\right]-E_{ \pm}+i \eta^{+}}
$$

with a positive infinitesimal $\eta^{+}$, the dispersion relation $E_{ \pm}= \pm|\phi(\mathbf{q})|$ and the wave function $|\psi\rangle_{ \pm}=\frac{\sqrt{2}}{2}\left(|\psi\rangle_{A} \pm \sqrt{\frac{\phi^{*}(\mathbf{q})}{\phi(\mathbf{q})}}|\psi\rangle_{B}\right)$, where the plus (minus) sign applies to the upper (lower) $\pi^{*}(\pi)$ band,

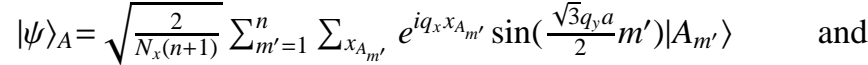

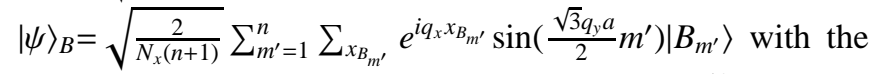
number of unit cells $N_{x}$ along the $x$-direction. ${ }^{19}$ A direct calculation yields expressions for the GF of an AGNR

$$
g_{a a}^{r}(\omega)=\frac{12 a}{N_{x}(n+1)} \frac{\omega-\left(\epsilon_{0}+s \Delta\right)+i \eta^{+}}{2 \pi} \int_{\frac{-\pi}{3 a}}^{\frac{\pi}{3 a}} d q_{x} \sum_{m, m^{\prime}=1}^{n} \frac{\sin ^{2}\left(\frac{m \pi}{n+1}\right) m^{\prime}}{\left[\omega-\left(\epsilon_{0}+s \Delta\right)+i \eta^{+}\right]^{2}-t^{2}\left[1+4 \cos ^{2} \frac{m \pi}{n+1}+4 \cos \frac{m \pi}{n+1} \cos \left(3 q_{x} / 2\right)\right]}(10)
$$

The exchange term splits the system into two separate spin subsystems. Therefore, the DOS, the linear conductance, and the current for spin-up and -down channels can be respectively obtained as

$$
\rho_{\uparrow(\downarrow)}(\omega)=-\frac{1}{\pi} \operatorname{Im} G_{a \uparrow \uparrow(\downarrow \downarrow)}^{r}(\omega),
$$




$$
\begin{gathered}
G_{\uparrow(\downarrow)}=\left.\frac{e^{2}}{h} X_{\uparrow \uparrow(\downarrow \downarrow)}(\omega)\right|_{\omega=E_{F}}, \\
I_{\uparrow(\downarrow)}(V)=\frac{e}{\hbar} \int_{-e V / 2}^{e V / 2} \frac{d \omega}{2 \pi}\left(f_{R}-f_{L}\right) X_{\uparrow \uparrow(\downarrow \downarrow)}(\omega),
\end{gathered}
$$

where $V$ is the applied bias voltage, and the matrix elements in Eqs. (11)-(13) are defined as

$$
G_{a}^{r}(\omega)=\sum_{\mathbf{q} \mathbf{q}^{\prime}} G_{\mathbf{q}^{\prime} a, \mathbf{q}^{\prime} a}^{r(a)}(\omega)=\left(\begin{array}{cc}
G_{a \uparrow}^{r}(\omega) & G_{a \uparrow \downarrow}^{r}(\omega) \\
G_{a \downarrow \uparrow}^{r \uparrow}(\omega) & G_{a \downarrow \downarrow}^{r \downarrow}(\omega)
\end{array}\right)
$$

and

$$
X(\omega)=\left[G_{a}^{r}(\omega)\left(R \boldsymbol{\Gamma}_{R} R^{\dagger}\right)\right] G_{a}^{a}(\omega) \boldsymbol{\Gamma}_{L}=\left(\begin{array}{ll}
X_{\uparrow \uparrow}(\omega) & X_{\uparrow \downarrow}(\omega) \\
X_{\uparrow \downarrow}(\omega) & X_{\downarrow \downarrow}(\omega)
\end{array}\right) .
$$

On the other hand, the STT is the time evolution rate of the total spin of left or right FM lead. ${ }^{26,27}$ Here, we ignored the interlayer exchange coupling, so the out-of-plane torque is zero. ${ }^{44}$ By means of the NGF method, the current-induced inplane STT along $x^{\prime}$-direction in the $\left(x^{\prime}, y^{\prime}\right)$ coordinate frame [see Fig. 1] exerting on the right FM lead can be obtained ${ }^{32}$

$$
\begin{aligned}
\tau_{R}^{x^{\prime}}= & \frac{1}{4 \pi} \int d \omega\left(f_{R}-f_{L}\right) \operatorname{Tr}\left[\mathrm{G}_{a}^{r}(\omega) \Gamma_{L} \mathrm{G}_{a}^{a}(\omega) R \Gamma_{R} R^{\dagger}\right. \\
& \left.\cdot\left(-\cos \theta \sigma_{x}+\sin \theta \sigma_{z}\right)\right],
\end{aligned}
$$

where $\sigma_{x}$ and $\sigma_{z}$ are the Pauli matrices.

\section{RESULTS AND DISCUSSIONS}

In what follows, we present some numerical examples of $\rho_{\uparrow(\downarrow)}, G_{\uparrow(\downarrow)}, G_{d, \uparrow(\downarrow)}$, TMR, and $\tau_{R}^{x^{\prime}}$ for the system with 7- or 8-AGNR at low temperatures according to Eqs. (11)-(16). In the calculation, the two FM leads are assumed to be made of the same materials, i.e., $p_{L}=p_{R}=p$, where $p_{L(R)}=\left(\Gamma_{L(R) \uparrow}-\right.$ $\left.\Gamma_{L(R) \downarrow}\right) /\left(\Gamma_{L(R) \uparrow}+\Gamma_{L(R) \downarrow}\right)$ is the polarization strength of the left (right) FM. Under the wide bandwidth approximation, $\Gamma_{L \uparrow(\downarrow)}=\Gamma_{R \uparrow(\downarrow)}=\Gamma_{0}(1 \pm p)$. The TMR is conventionally defined as TMR $=\left(I_{P}-I_{A P}\right) / I_{P}$, where $I_{P}$ and $I_{A P}$ are the total current for the parallel and antiparallel configuration, respectively. The coupling $\Gamma_{0}$ between the AGNR and leads without internal magnetization is taken as the energy unit. The chemical potentials for the two FM leads are set as $\mu_{L, R}=E_{F} \pm 0.5 \mathrm{eV}$ with the Fermi energy $E_{F}=0$. The on-site energy $\epsilon_{0}=0$, and $\Gamma_{0}=t$ because that they are should be in the same order. ${ }^{45}$

\section{A. Spin-dependent transport}

In Fig. 2, we present the spin-dependent DOS versus energy $\omega$ (in units of $\left.\Gamma_{0}\right)$ in parallel configuration $(\theta=0)$ with exchange splitting $\Delta=0.05$ and different polarization strength $p$ for the system with 7- and 8-AGNR, respectively. In the case of bare AGNR $(\Delta=\Gamma=0)$, a zero value plateau in DOS for 7AGNR appears symmetrically with respect to the Fermi level
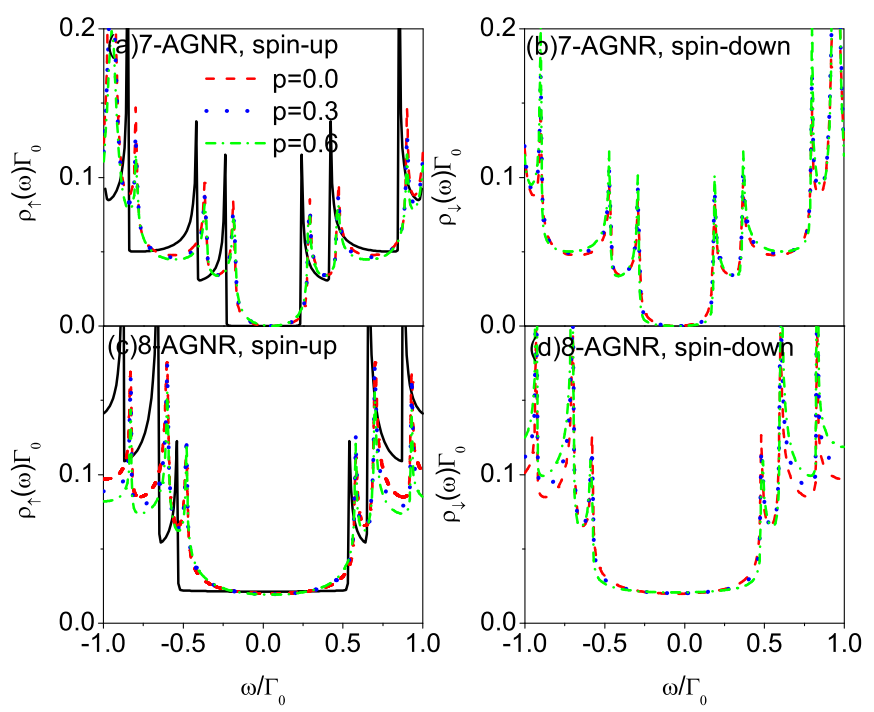

FIG. 2: (Color online) DOS for spin-up [(a) and (c)], and -down [(b) and (d)] channels as a function of energy $\omega$ (in units of $\Gamma_{0}$ ) with different polarization $p$, where the parameters are taken as $\epsilon_{0}=0, \theta=0$ and $\Delta=0.05$. For comparison, the DOS for bare $\left(\Delta=\Gamma_{0}=0\right) 7$-AGNR (a) and 8-AGNR (c) is shown in (dark) solid lines.

[see (dark) solid line in Fig. 2(a)] due to the presence of the energy gap for semiconducting 7-AGNR, while for 8-AGNR a nonzero symmetrical plateau with respect to the Fermi level appears [see (dark) solid line in Fig. 2(c)] because of the gapless energy band in metallic 8-AGNR. However, when a magnetic deposition is applied, the positions of plateaus for both 7-AGNR and 8-AGNR systems shift with the exchange field strength $\Delta$ : the spin-up (-down) DOS deviates the origin point by the magnitude of $\Delta$ towards the positive (negative) direction of the energy. The reason for this phenomenon is that the exchange interaction in the magnetic deposition behaves as an effective in-plane magnetic field, and acts on the electrons in AGNRs, thus leading to the shift of the energy level related to the electron spin. For larger $\omega, \rho_{\sigma}$ shows an oscillation behavior with sharp peaks, which indicates the buildup of Van Hove singularities (VHSs) at subband edge. Additionally, we notice that as $p$ increases from zero [(red) dashed lines for normal leads] to 0.6 [(green) dash-dotted lines] in Fig. 2, both 7- and 8-AGNR systems are almost immune from $p$, which is different from the bulk graphene tunneling junction. ${ }^{39}$ This stems from the fact the VHSs induced by the edge effect of AGNRs are independent on $p$.

Figure 3 shows the spin-dependent linear conductance (in units of $e^{2} / h$ ) versus exchange splitting $\Delta$ (in units of $\Gamma_{0}$ ) with different polarization $p$ in parallel alignment at the Fermi energy for the system with 7- and 8-AGNR, respectively. It is seen that for both 7-AGNR and 8-AGNR systems, $G_{\uparrow}$ increases [see Figs. 3(a) and 3(c)] while $G_{\downarrow}$ decreases [see Figs. $3(\mathrm{~b})$ and 3(d)] with the increase of polarization $p$, which is due to the conventional spin-valve effect in the magnetic tunneling junction. Namely, with increasing $p$, the proportion of spin-up electrons increases and that of spin-down electrons decreases in AGNRs. Consequently, it becomes easier for spin-up electrons to tunnel through the barrier, but harder for spin-down 

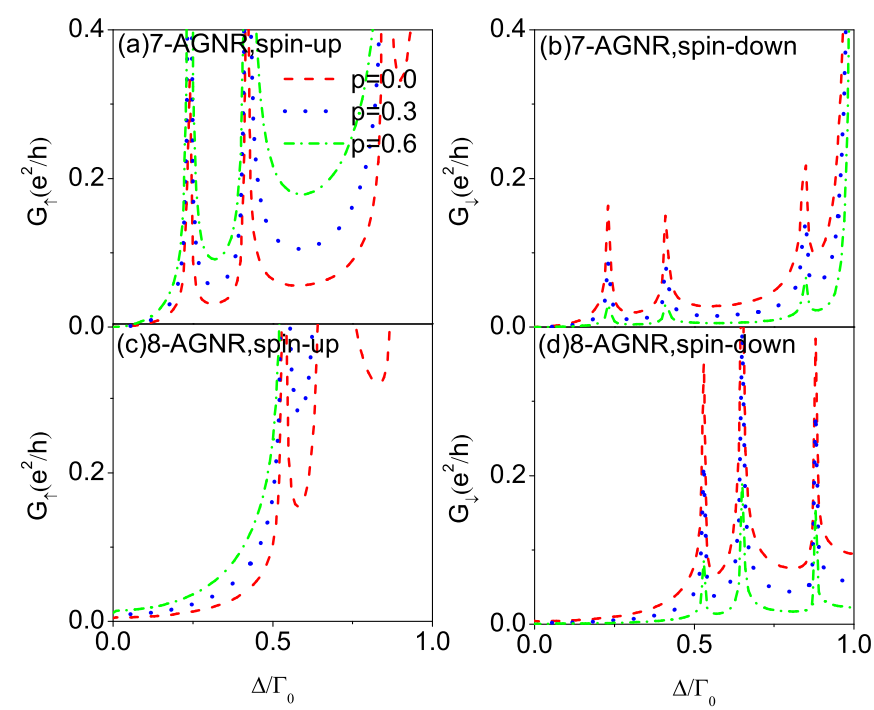

FIG. 3: (Color online) The linear conductance as a function of exchange splitting $\Delta$ with different polarization $p$ for spin-up [(a) and (c)], and spin-down [(b) and (d)] channels. The other parameters are the same as those of Fig. 2.
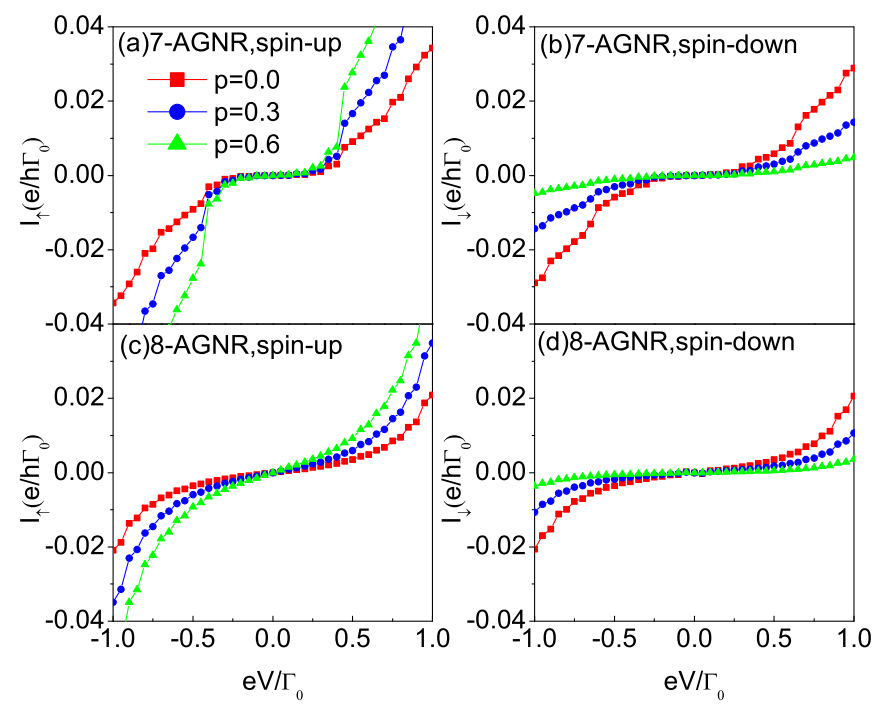

FIG. 4: (Color online) The I-V curve with different polarization $p$ for spin-up [(a) and (c)], and spin-down [(b) and (d)] channels. The parameters are the same as those of Fig. 2.

ones due to the presence of the inverse spin-direction for spindown electrons in the tunneling process, thus we conclude that $G_{\uparrow}$ increases while $G_{\downarrow}$ decreases with the increase of $p$. In addition, it is found that for the small $\Delta$ (below the full spin polarization ${ }^{46}$ ), the conductance value is zero for 7-AGNR system, while nonzero constant for 8-AGNR system. This is related to the presence (absence) of energy gap in semiconducting 7-AGNR (metallic 8-AGNR). For a larger $\Delta$ (above the full spin polarization), the conductance for either 7- or 8AGNR system displays an oscillation enhancement behavior with shape peaks, which stems from the resonant tunneling through different subbands due to the edge effect. ${ }^{47,48}$

The bias voltage dependence of current (in units of $e / h \Gamma_{0}$ )
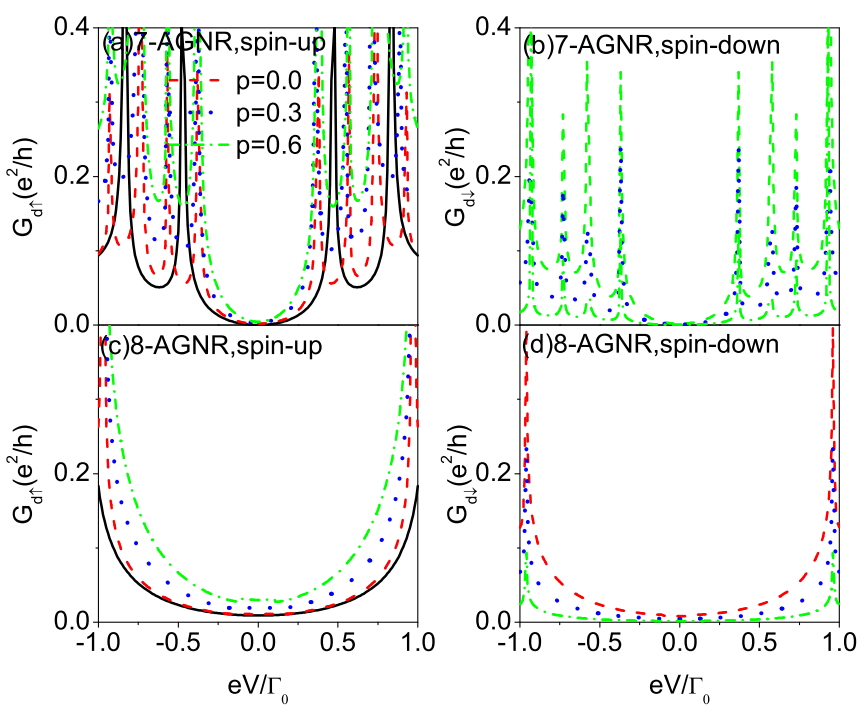

FIG. 5: (Color online) The differential conductance as a function of bias $V$ with different polarization $p$ for spin-up [(a) and (c)], and spin-down [(b) and (d)] channels. The other parameters are the same as those of Fig. 2. For comparison, the differential conductance for 7- and 8-AGNR with $\Delta=p=0$ is also shown in (dark) solid lines.

for the 7-AGNR and 8-AGNR systems with different polarization $p$ under the parallel configuration is demonstrated in Fig. 4. It is clearly seen that the current has a step-like structure as a function of bias voltage, which results from the constant DOS at the Fermi energy and resonance due to VHSs. In particular, the dependence of the current on the polarization $p$ exhibits different behaviors for the 7-AGNR and 8-AGNR systems. With increasing $p$, the current increases in the entire bias voltage for the 8-AGNR system, while for the 7-AGNR system, the current rises only in the large bias, and almost remains zero at small bias voltage. The explanation for this phenomenon is as follows: the DOS for the 7-AGNR system vanishes near the Fermi level, thus the 7-AGNR system resembles a insulator-like barrier at the low energy. In this case, the electrons will difficultly tunnel through the 7-AGNR system at low bias.

In Fig. 5, we demonstrate the dependence of differential conductance $G_{d}=d I / d V$ (in units of $e^{2} / h$ ) on the bias voltage for the system with different polarization $p$ under the parallel configuration. For comparison, we plot the differential conductance of the 7- and 8-AGNR systems for $\Delta=p=0$ in (dark) solid lines. The differential conductance exhibits the successive oscillation peaks corresponding to the resonant tunneling through the edge-induced subbands. In addition, we find that there exists a respective plateau structure at small bias for the 7- and 8-AGNR systems. In the range of the plateau, the conductance approaches to zero for the 7-AGNR system, while a finite constant for 8-AGNR system. An interesting characteristic is that the plateau structure for the 8-AGNR system is broader than that for the 7-AGNR system since 8-AGNR has a larger interval between the Fermi energy and the lowest subband [see Fig. 2]. When $\Delta$ is applied, the peak splits into two peaks located at the two sides of the original peak for both 7-AGNR and 8-AGNR systems, and the position of the peaks 


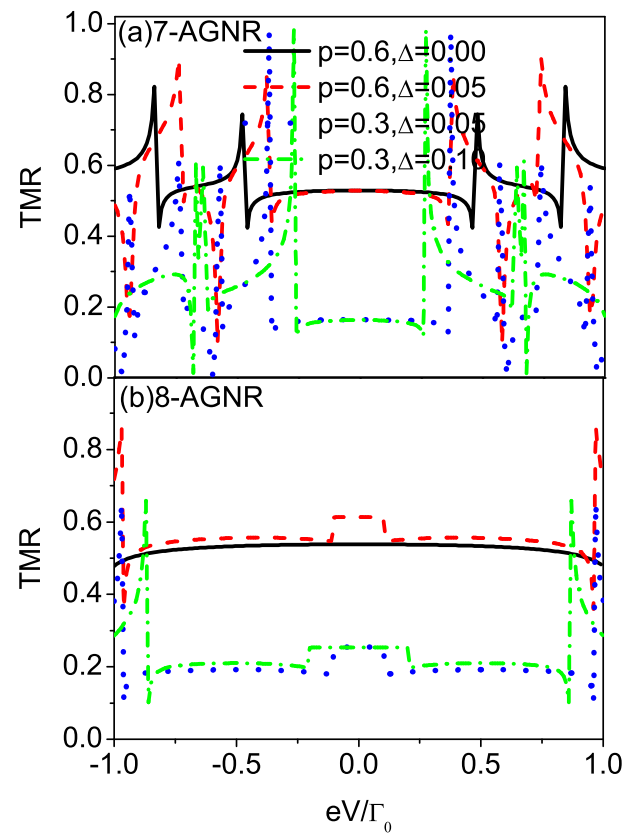

FIG. 6: (Color online) The dependence of TMR on bias $V$ for (a) 7AGNR, (b) 8-AGNR system with different polarization $p$ and splitting $\Delta$.

depends on the magnitude of the exchange splitting. This behavior is due to the exchange splitting of the edge-induced subbands. From Fig. 5, $G_{d \uparrow \uparrow}$ increases while $G_{d, \downarrow}$ decreases for both 7- and 8-AGNR systems with increasing $p$, which is a typical spin-valve effect.

Figure 6 shows the TMR versus the bias voltage $V$ for 7 and 8-AGNR systems with different polarization $p$ and exchange splitting $\Delta$. Firstly, it is noted that the TMR for the 7and 8-AGNR systems has a high value relative to the conventional magnetic junction; a pronounced plateau for 7- and 8AGNR systems appears at lower bias due to the constant DOS near the Fermi energy [see Fig. 2]. These results are quite consistent with the previous studies. ${ }^{39,44}$ For high bias, the TMR exhibits the successive oscillation peaks resided near the edgeinduced subbands. The amplitude of these peaks can even approach to 1 indicating an perfect spin-valve effect. A high TMR for the system with an AGNR has also been obtained in Refs. $[49,50]$. It should be pointed out that the result here is different from that of Ref. [23] where the TMR for the AGNRs systems is very small in the vicinity of the Fermi energy. The plateau structure for 8-AGNR system is systematically broader than that corresponding to 7-AGNR system, which may be very useful to overcome a well-known shortcoming of MRAM, which is the large decrease in TMR with applied bias voltage. Interestingly, the increase of $\Delta$ suppresses the amplitude of this structure for 7-AGNR system. However, the TMR is enhanced within bias range from $-\Delta$ to $\Delta$ for 8-AGNR system. This is caused by the exchange splitting influence on the DOS as mentioned in Fig. 2. The results are quantitatively different from our previous work for FM/graphene/FM system due to the finite size for AGNRs. ${ }^{16,39}$ When the polarization changes to $p=0.3$, as shown by the (blue) dotted lines
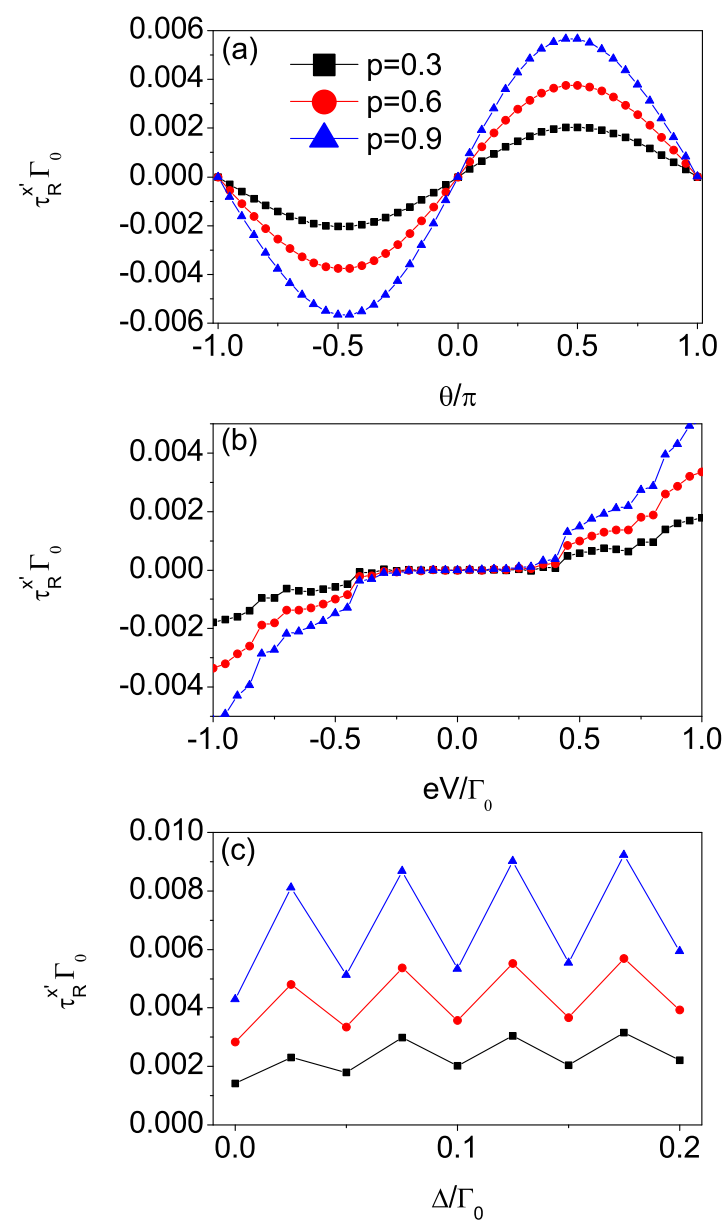

FIG. 7: (Color online) The dependence of current-induced STT for 7-AGNR on (a) relative orientation angle $\theta$ at the bias voltage $V=0.5 \Gamma_{0} / e$, (b) bias voltage $V$ at $\theta=\pi / 3$, and (c) exchange splitting $\Delta$ at $\theta=\pi / 3$ and $V=0.5 \Gamma_{0} / e$ with different polarization $p$. The other parameters are the same as in Fig. 2.

and (green) dash-dotted lines in Fig. 6, the TMR for both 7-AGNR and 8-AGNR systems becomes obviously smaller because the leads become less spin-polarized with decreasing $p .^{51}$ This result is different from Ref. [15], where the TMR is nearly independent of the electronic details of the leads. We speculate that the structure in the Ref. [15] is very different from the structure considered here. Our results suggest that it is more favorable to fabricate a graphene-based spin-valve device by using a metallic AGNR.

\section{B. Spin transfer torque}

Figures 7 and 8 show the current-induced STT $\left(\tau_{R}^{x^{\prime}}\right)$ as functions of angular $\theta$, bias voltage and exchange splitting strength for 7-AGNR and 8-AGNR systems with different polarization $p$. It is seen that $\tau_{R}^{x^{\prime}}$ versus the angle $\theta$ shows a sine-like behavior for 7-AGNR and 8-AGNR systems, which is in line with the previous findings. ${ }^{28,39} \mathrm{~A}$ similar result has also been obtained for a FM/NM/FM trilayer system discussed in Ref. [52], although the transport mechanisms are different. The 

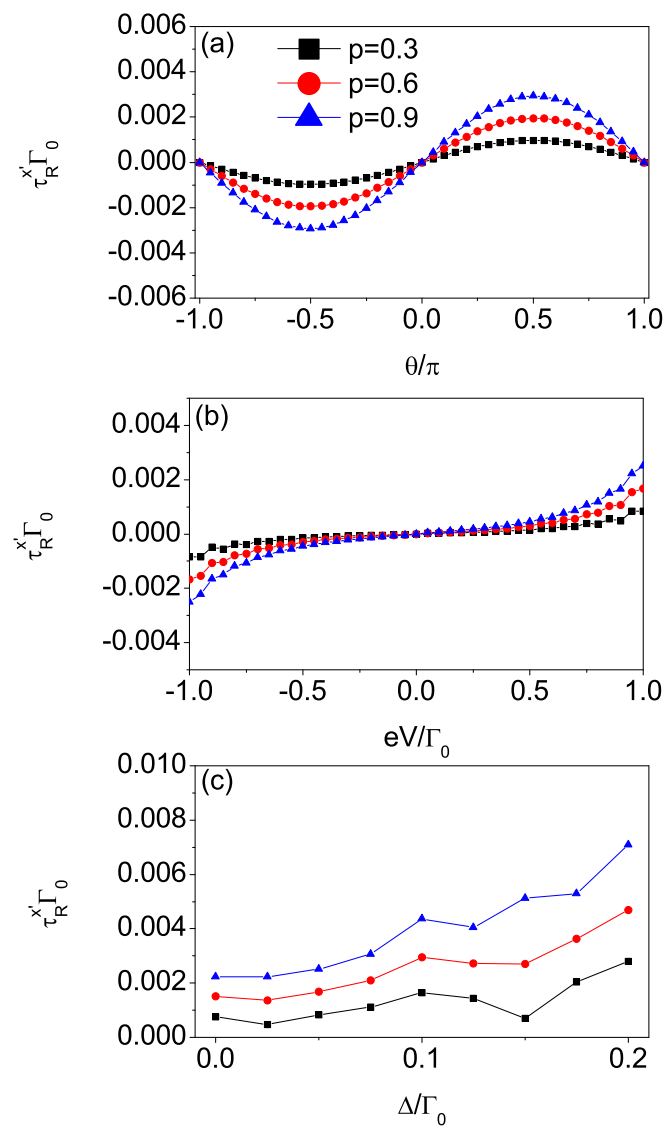

FIG. 8: (Color online) The dependence of current-induced STT for 8-AGNR on (a) relative orientation angle $\theta$ at the bias voltage $V=0.5 \Gamma_{0} / e$, (b) bias voltage $V$ at $\theta=\pi / 3$, and (c) exchange splitting $\Delta$ at $\theta=\pi / 3$ and $V=0.5 \Gamma_{0} / e$ with different polarization $p$. The other parameters are the same as in Fig. 2.

present result can be easily understood because the spin torque is proportional to $\mathbf{S}_{\mathbf{R}} \times\left(\mathbf{S}_{\mathbf{L}} \times \mathbf{S}_{\mathbf{R}}\right)$, where $\mathbf{S}_{\mathbf{L}}$ and $\mathbf{S}_{\mathbf{R}}$ are the spin moments of the left and right FMs, respectively. Thus we can conclude that $\tau_{R}^{x^{\prime}}$ vanishes when the relative alignment of magnetization of the two FM leads is parallel $(\theta=0)$ or antiparallel $(\theta=\pi)$. Furthermore, the STT for 7-AGNR system is quantitatively larger than that for 8-AGNR system, suggesting that a semiconducting AGNR is a better choice for a currentcontrolled magnetic memory element.

In addition, it is evident that with increasing polarization $p$, the STT is enhanced, as displayed in Fig. 7(a) and Fig. 8(a). This is in line with the statement that $\tau_{R}^{x^{\prime}}$ is proportional to the polarization strength of $\mathrm{FM}^{28}$ Similar result is obtained in the conventional spin-valve device. ${ }^{26}$ From Figs. 7(b) and 8(b), one can notice the step-like feature in the STT as a function of bias voltage for both 7- and 8-AGNR systems, which is reminiscent of the current versus the bias. The reason is that the in-plane $\tau_{R}^{x^{\prime}}$ is proportional to the difference of spinpolarized current $\left(I^{s}(0)-I^{S}(\pi)\right),{ }^{28,31}$ where $I^{s}(\theta)=I_{\uparrow}(\theta)-I_{\downarrow}(\theta)$ is the spin-current densities for the angle $\theta$. In practice, $I^{s}(\pi)$ (not shown here) for both 7- and 8-AGNR systems vanishes identically due to the absence of minority states available for tunneling. Thereby, the bias dependence of $\tau_{R}^{x^{\prime}}$ remains the similar feature to that of $I^{s}(0)$. This is also the reason why the anomalous bias dependence of the STT has not been predicted in this paper according to Ref. [31], where anomalous bias dependence of the STT only appears in the case of $I^{S}(0) \leq I^{s}(\pi)$. Another reason for this is that the result is obtained here under the wide bandwidth approximation. From Figs. 7(c) and $8(\mathrm{c})$, it can be found that $\tau_{R}^{x^{\prime}}$ with the exchange splitting $\Delta$ displays a oscillation behavior for the 7-AGNR and 8-AGNR systems. This result stems from the fact that the exchange splitting shifts the VHSs [cf. Figs. 2(a) and (c)], thus leads to the edge-induced subband crossing of the bias windows causing the tunneling resonance.

\section{SUMMARY AND CONCLUSION}

In conclusion, we have demonstrated the effects of the AGNR width, the polarization strength of FM leads with arbitrary polarization directions and the exchange splitting on the spin-resolved transport properties for both semiconducting and metallic AGNRs. In contrast to other related theoretical works, the leads in our system are not supposed to be two-dimensional of honeycomb- or square-lattice type, but is more realistical modeled as three-dimensional semi-infinite FM slabs. By means of Keldysh NGF method, it is found that the spin-resolved transport property for the system depends sensitively on both the AGNR type and the polarization strength of FM leads. Around zero bias the TMR versus bias voltage possesses a pronounced plateau structure for both semiconducting 7-AGNR and metallic 8-AGNR systems without exchange splitting. This phenomenon may be very useful to overcome a well-known shortcoming of MRAM, which is the large decrease in TMR with applied bias voltage. Remarkably, the plateau structure for 8-AGNR system is much broader than that for 7-AGNR system. Interestingly, the increase of the exchange splitting $\Delta$ suppresses the amplitude of this structure for 7-AGNR system. However, the TMR is enhanced at bias that ranged from $-\Delta$ to $\Delta$ for 8-AGNR system. So it may be useful in the design of spin-valve device and graphene nanoribbons-based spintronic devices by using a metallic AGNR. In addition, the current-induced STT for 7-AGNR system is systematically larger that that for 8AGNR system, which may be more favorable to fabricate a current-controlled magnetic memory element by using a semiconducting AGNR. As a development of graphene fabrication technology, several tens of nanometer wide GNRs can be obtained by patterning graphene into a narrow ribbon, and the large exchange splitting can also be realizable in GNRs, thus the model considered in this paper may have potential application in spintronic devices.

\section{Acknowledgments}

This work was supported by the National Natural Science Foundation of China (Grant Nos. 10974052 and 10574042), the Program for Changjiang Scholars and Innovative Research Team in University (PCSIRT, No. IRT0964), and the Scien- 
* Electronic address: ghzhou@ @unnu.edu.cn

1 S. A. Wolf, D. D. Awschalom, R. A. Buhrman, J. M. Daughton, S. von Molnár, M. L. Roukes, A. Y. Chtchelkanova, and D. M. Treger, Science 294, 1488 (2001)

2 Daniel Huertas-Hernando, F. Guinea, and Arne Brataas, Phys. Rev. B 74, 155426 (2006).

${ }^{3}$ Nikolaos Tombros, Csaba Jozsa, Mihaita Popinciuc, Harry T. Jonkman, and Bart J. van Wees, Nature (London) 448, 571 (2007).

4 Sungjae Cho, Yung-Fu Chen, and Michael S. Fuhrerb, Appl. Phys. Lett. 91, 123105 (2007).

5 Wei Han, K. Pi, W. Bao, K. M. McCreary, Yan Li, W. H. Wang, C. N. Lau, and R. K. Kawakami, Appl. Phys. Lett. 94, 222109 (2009).

${ }^{6}$ C. Józsa, M. Popinciuc, N. Tombros, H. T. Jonkman, and B. J. van Wees, Phys. Rev. B 79, 081402(R) (2009).

7 Tamalika Banerjee, Wilfred G. van der Wiel, and Ron Jansen, Phys. Rev. B 81, 214409 (2010).

8 Wei Han, K. Pi, K. M. McCreary, Yan Li, Jared J. I. Wong, A. G. Swartz, and R. K. Kawakami, Phys. Rev. Lett. 105, 167202 (2010).

9 Jeroen B. Oostinga, Benjamin Sacépé, Monica F. Craciun, and Alberto F. Morpurgo, Phys. Rev. B 81, 193408 (2010).

${ }^{10}$ Ernie W. Hill, Andre K. Geim, Konstantin Novoselov, Frederik Schedin, and Peter Blake, IEEE Trans. Magn. 42, 2694

11 W. H. Wang, K. Pi, Y. Li, Y. F. Chiang, P. Wei, J. Shi, and R. K. Kawakami, Phys. Rev. B 77, 020402(R) (2008).

12 Håvard Haugen, Daniel Huertas-Hernando, and Arne Brataas, Phys. Rev. B 77, 115406 (2008).

13 E. H. Hwang and S. Das Sarma, Phys. Rev. B 80, 075417 (2009).

14 M. Khodas, I. A. Zaliznyak, and D. E. Kharzeev, Phys. Rev. B 80, 125428 (2009).

15 L. Brey, and H. A. Fertig, Phys. Rev. B 76, 205435 (2007).

16 Kai-He Ding, Zhen-Gang Zhu, and Jamal Berakdar, Phys. Rev. B 79, 045405 (2009).

17 Kyoko Nakada, Mitsutaka Fujita, Gene Dresselhaus, and Mildred S. Dresselhaus, Phys. Rev. B 54, 17954 (1996).

18 Young-Woo Son, Marvin L. Cohen, and Steven G. Louie, Phys. Rev. Lett. 97, 216803 (2006).

19 Huaixiu Zheng, Z. F. Wang, Tao Luo, Q. W. Shi, and Jie Chen, Phys. Rev. B 75, 165414 (2007).

${ }^{20}$ Woo Youn Kim, and Kwang S. Kim, Nat. Nanotech. 3, 408 (2008).

21 F. Muñoz-Rojas, J. Fernández-Rossier, and J. J. Palacios, Phys. Rev. Lett. 102, 136801 (2009).

22 Young-Woo Son, Marvin L. Cohen and Steven G. Louie, Nature 444, 347 (2006).

23 Jiang-chai Chen, Shu-guang Cheng, Shun-Qing Shen, and Qingfeng Sun, J. Phys.: Condens. Matter 22, 035301 (2010); Ying-Tao Zhang, Hua Jiang, Qing-feng Sun, and X. C. Xie, Phys. Rev. B 81, 165404 (2010).

${ }^{24}$ Rui Qin, Jing Lu, Lin Lai, Jing Zhou, Hong Li, Qihang Liu, Guangfu Luo, Lina Zhao, Zhengxiang Gao, Wai Ning Mei, and Guangping Li, Phys. Rev. B 81, 233403 (2010).

25 Jingwei Bai, Rui Cheng, Faxian Xiu, Lei Liao, Minsheng Wang, Alexandros Shailos, Kang L. Wang, Yu Huang, and Xiangfeng Duan, Nat. Nanotech. 5, 655 (2010).

26 J. C. Slonczewski, J. Magn. Magn. Mater. 159, L1 (1996).

27 L. Berger, Phys. Rev. B 54, 9353 (1996).

28 J. C. Slonczewski, Phys. Rev. B 71, 024411 (2005).
${ }^{29}$ Christian Heiliger, and M. D. Stiles, Phys. Rev. Lett. 100, 186805 (2008).

30 Shuai Wang, Ling Tang, and Ke Xia, Phys. Rev. B 81, 094404 (2010).

31 Ioannis Theodonis, Nicholas Kioussis, Alan Kalitsov, Mairbek Chshiev, and W. H. Butler, Phys. Rev. Lett. 97, 237205 (2006); Alan Kalitsov, Mairbek Chshiev, Ioannis Theodonis, Nicholas Kioussis, and W. H. Butler, Phys. Rev. B 79, 174416 (2009).

${ }^{32}$ Hai-Feng $\mathrm{Mu}$, Gang Su, and Qing-Rong Zheng, Phys. Rev. B 73, 054414 (2006).

33 Jack C. Sankey, Yong-Tao Cui, Jonathan Z. Sun, John C. Slonczewski, Robert A. Buhrman, and Daniel C. Ralph, Nat. Phys. 4, 67 (2008)

34 Z. Li, S. Zhang, Z. Diao, Y. Ding, X. Tang, D. M. Apalkov, Z. Yang, K. Kawabata, and Y. Huai, Phys. Rev. Lett. 100, 246602 (2008).

35 E. B. Myers, D. C. Ralph, J. A. Katine, R. N. Louie, and R. A. Buhrman, Science 285, 867 (1999).

36 J. A. Katine, F. J. Albert, R. A. Buhrman, E. B. Myers, and D. C. Ralph, Phys. Rev. Lett. 84, 3149 (2000).

37 G. D. Fuchs, N. C. Emley, I. N. Krivorotov, P. M. Braganca, E. M. Ryan, S. I. Kiselev, J. C. Sankey, D. C. Ralph, R. A. Buhrman, and J. A. Katine, Appl. Phys. Lett. 85, 1205 (2004); G. D. Fuchs, J. A. Katine, S. I. Kiselev, D. Mauri, K. S. Wooley, D. C. Ralph, and R. A. Buhrman, Phys. Rev. Lett. 96, 186603 (2006).

${ }^{38}$ S. S. P. Parkin, K. P. Roche, M. G. Samant, P. M. Rice, R. B. Beyers, R. E. Scheuerlein, E. J. O'Sullivan, S. L. Brown, J. Bucchigano, D. W. Abraham, Yu Lu, M. Rooks, P. L. Trouilloud, R. A. Wanner, and W. J. Gallagher, J. Appl. Phys. 85, 5828 (1999).

39 Benhu Zhou, Xiongwen Chen, Haiyan Wang, Kai-He Ding, and Guanghui Zhou, J. Phys.: Condens. Matter 22, 445302 (2010).

${ }^{40}$ Hartmut J. W. Haug, and Antti-Pekka Jauho, Quantum Kinetics in Transport and Optics of Semiconductors (Springer, Berlin, 1998).

41 Yigal Meir, and Ned S. Wingreen, Phys. Rev. Lett. 68, 2512 (1992).

42 Yugui Yao, Fei Ye, Xiao-Liang Qi, Shou-Cheng Zhang, and Zhong Fang, Phys. Rev. B 75, 041401(R) (2007).

43 M. P. López-Sancho, and M. C. Muñoz, Phys. Rev. B 83, 075406 (2011).

44 P. Bruno, Phys. Rev. B 49, 13231 (1994).

45 A. Saffarzadeh, and M. Ghorbani Asl, Eur. Phys. J. B 67, 239 (2009).

46 The full spin polarization, namely, the graphene fully ferromagnetic depends on the magnitude of the exchange splitting, which depends on the graphene carrier density (Ref. 13). It is estimated that the values of the exchange splitting to make the graphene fully ferromagnetic are in the range of $0.05-0.2 \mathrm{eV}$ when the carrier density is in the range of $10^{11}-10^{12}$ (Refs. 12 and 13).

47 J. U. Nöckel, Phys. Rev. B 46, 15348 (1992).

48 M. L. Ladrón de Guevara, and P. A. Orellana, Phys. Rev. B 73, 205303 (2006).

49 S. Krompiewski, Phys. Rev. B 80, 075433 (2009).

50 S. Honda, A. Yamamura, T. Hiraiwa, R. Sato, J. Inoue, and H. Itoh, Phys. Rev. B 82, 033402 (2010).

51 M. Julliere, Phys. Lett. A 54, 225 (1975).

52 Xavier Waintal, Edward B. Myers, Piet W. Brouwer, and D. C. Ralph, Phys. Rev. B 62, 12317 (2000). 